\title{
Limitations of smoothening functions for automotive vibro-impact problems
}

\author{
Zhiwei Zhang $^{\mathrm{a}, *}$, Rajendra Singh ${ }^{\mathrm{b}}$ and Ashley R. Crowther ${ }^{\mathrm{b}}$ \\ ${ }^{\mathrm{a}}$ Wolfson School of Mechanical and Manufacturing Engineering, Loughborough University, Leicestershire LE11 \\ $3 T U, U K$ \\ ${ }^{\mathrm{b}}$ Acoustics and Dynamics Laboratory, Mechanical Engineering Department, The Ohio State University, Columbus, \\ OH, USA
}

Received 13 February 2010

Revised 31 May 2010

\begin{abstract}
Nonlinear torsional models are used to analyze automotive transmission rattle problems and find solutions to reduce noise, vibration and dynamic loads. The torsional stiffness and inertial distribution of such systems show that the underlying mathematical problem is numerically stiff. In addition, the clearance nonlinearities in the gear meshes introduce discontinuous functions. Both factors affect the efficacy of time domain integration and smoothening functions are widely used to overcome computational difficulties and improve the simulation. In this paper, alternate smoothening functions are studied for their influence on the numerical solutions and their impact on global convergence and computation times. In particular, four smoothening functions (arctan, hyperbolic-cosine, hyperbolic-tan and quintic-spline) are applied to a five-degree-of-freedom generic torsional system with two backlash (clearance) elements. Each function is assessed via a global convergence metric across an excitation map (a design of experiment). Regions of the excitation map, along with multiple solutions, are studied and the implications to assessing convergence are critically examined. It is observed that smoothening functions do not lead to better convergence in many cases. The smoothening parameter needs to be carefully selected, or over-smoothened solutions may be found. The system studied is representative of a typical automotive rattle problem and it was found that benefits were limited from applying such smoothening functions.
\end{abstract}

Keywords: Vibro-impact systems, non-smooth dynamics, computational issues, smoothening functions, automotive gear rattle

\section{Nomenclature}

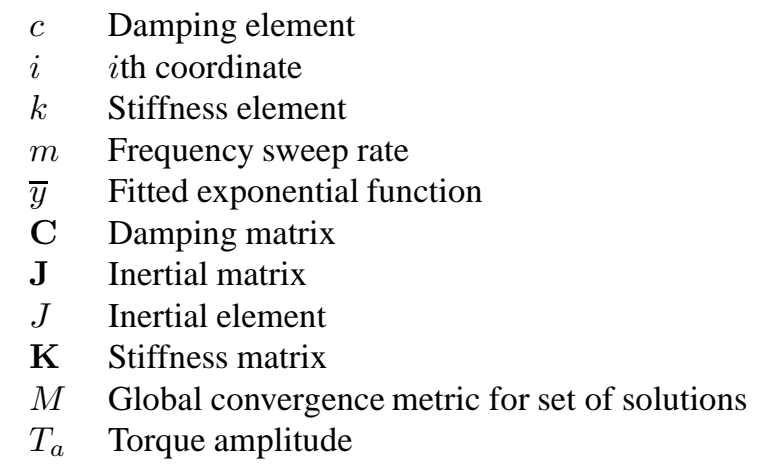

${ }^{*}$ Corresponding author. E-mail: z.zhang@lboro.ac.uk. 


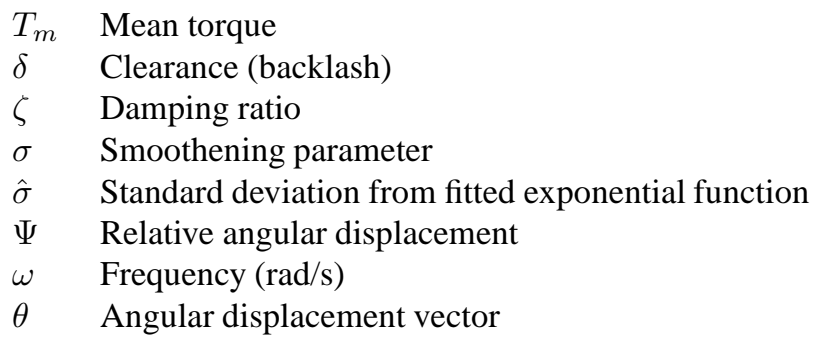

\section{Introduction}

The vibro-impacts in the automotive driveline affect the perceived quality of a vehicle when a rattling noise is audible within the passenger compartment. The noise source is the impacting gears responding to harmonic excitations from engine, typically under low mean load and the non-linear behaviour can be strong in certain operating regimes. The impacts occur as most transmissions have many clearance elements in the gear meshes which can be described with a contact stiffness and clearance discontinuity. Simulation of such non-smooth systems pose challenges in numerical analysis as described by [1-4]. When using ordinary differential equation solvers, such as Runge-Kutta routines, systems with discontinuous stiffness are typically handled two ways. They either are set to solve explicitly until a discontinuity and then stop with a restart after the parameter is set on the other side of the boundary, or alternatively integrate continuously across the boundary. Both methods reduce the order of accuracy of the solution and slow down variable step integrators significantly. An investigation into the application of various solvers to a gear rattle formulation has been published in the literature [5] and the authors recommended further investigation [5]. It is common to "smoothen" the discontinuities with a continuously differentiable function [6], or "smoothening function" which approximates the absolute function and renders the discontinuity less severe. This is expected to speed up the solution but the effect of such smoothening on the dynamic response or on the convergence of solutions has rarely been reported in the literature. In [7], arctan and quintic spline smoothening functions were applied to a generic automotive vibro-impact system and assessed for their effectiveness. It was found that the functions did not improve convergence, however for some excitations multiple solutions are possible and assessing convergence is then difficult as the system can move from one solution regime to another under a small perturbation in the parameter.

In this paper, four smoothening functions are evaluated for convergence and computational efficiency across a prescribed excitation region. Importantly, the region is first confirmed to exhibit just one type of solution. Further examination concerns the possibility of over-smoothening and selecting the magnitude of the smoothening parameter. The paper suggests a methodology that can be applied by researchers to carefully assess their formulation before proceeding with numerical studies of vibro-impact problems.

\section{A generic powertrain with clearances: Example case}

To study automotive vibro-impact problem a generic torsional model that is representative of the powertrain is selected by picking an appropriate set of eigenvalues, vectors and excitation sources. A model (Fig. 1) is formulated as $\mathbf{J} \ddot{\theta}+\mathbf{C} \dot{\theta}+\mathbf{K}(\theta) \theta(t)=\mathbf{T}(t, \theta)$. This system is excited by $T_{e}=T_{m}+T_{a} \sin \omega t$, which simplifies the torque from the combustion engine to a mean and one oscillatory component. Clearances in Fig. 1 are prescribed to locations $k_{23}$ and $k_{34}$ and are representative of transmission and driveline gear and spline backlashes. See the nomenclature section for definitions. For the non-smoothened case the clearance is modelled using the piecewise characteristics described below, see [7,8] for further details on the formulation. Note $\psi_{i, i+1}=\theta_{i}-\theta_{i+1}$, and represents relative angular displacement across the clearance. The stiffness elements are dependent on the relative displacements between the inertias and the clearance values.

$$
k_{i, i+1}= \begin{cases}k_{i, i+1} & \left|\Psi_{i, i+1}\right| \geqslant 0.5 \delta_{i, i+1} \\ 0 & \left|\Psi_{i, i+1}\right|<0.5 \delta_{i, i+1}\end{cases}
$$




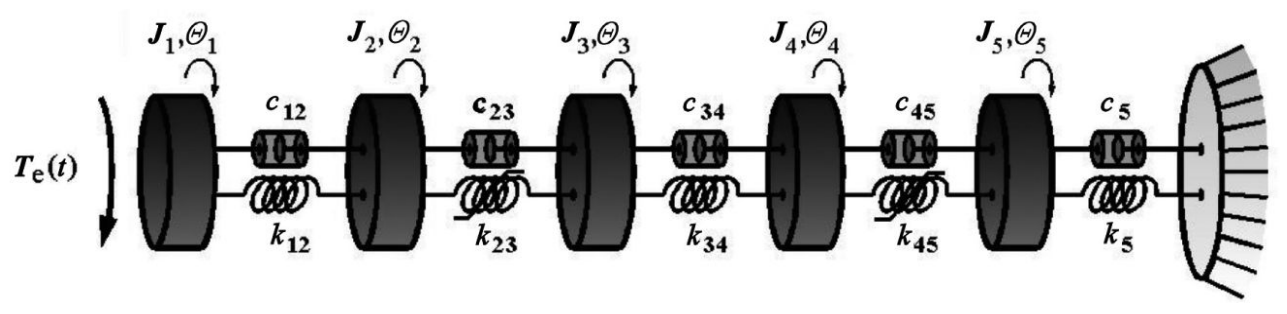

Fig. 1. Five DOF model of the generic torsional system. The elements $\mathrm{k}_{23}$ and $\mathrm{k}_{45}$ have been designated as typical locations for clearances and could represent, for example, the transmission and axle clearances.

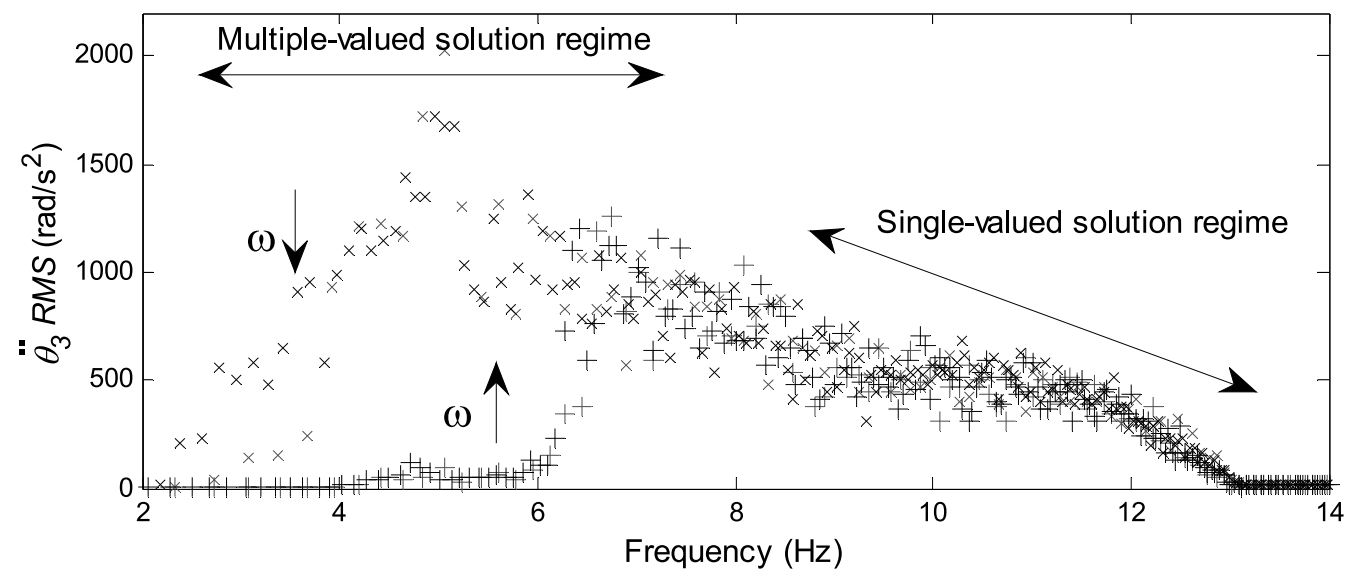

Fig. 2. Non-linear response map for frequency sweeps with a chirp signal with $m=0.5 \mathrm{Hzs}^{-1}$ (red cross symbol) and $1 \mathrm{Hzs}{ }^{-1}$ (black plus symbol).

\section{Nature of solutions}

\subsection{Non-linear response map}

The following methods or solutions are of interest: a) those that find the threshold of entering the clearance, which may be an analytical linear system; b) those for simplified formulations of the non-linear system semi-analytically solved by methods such as harmonic balance, typically of few degrees-of-freedom [9,10]; c) steady state numerical solutions for given excitations and d) transient numerical solutions with swept sinusoidal or chirp signal. Each method or solution is useful in understanding the phenomena and/or driveline design. The last approach (d) is applied here to show the nature of the solutions.

For the dynamic model of Fig. 1, four frequency sweeps are performed using the chirp function at two rates, $m=0.5$ and $1 \mathrm{Hzs}^{-1}$, from 2 to $14 \mathrm{~Hz}$ and for both rising and falling cases. A time windowed root-mean-square acceleration is chosen as a metric for rattle intensity, the window has the width of several periods of the excitation function (see [7] for further explanation). Figure 2 plots the rattle intensity metric for versus frequency for $\theta_{3}$. Note that the scatter in the response map is due to its construction from a transient solution. For rising frequency the system moves from no impacts to single-sided impacts at around $4 \mathrm{~Hz}$ and a small nonlinear resonance lies near $4.8 \mathrm{~Hz}$. A sharp increase in magnitude occurs at approximately $6 \mathrm{~Hz}$ as the system moves to double-sided impact behaviour $(6-7 \mathrm{~Hz})$; the transition frequency is dependent on sweep rate. From $7-13 \mathrm{~Hz}$ the single sided impacts are much harsher and above $13 \mathrm{~Hz}$ there are no impacts. (Note that the sweeps were not run to $50 \mathrm{~Hz}$, the next linear resonance, where additional regions of impacts occur). For falling frequency, the system moves from no impacts to single-sided impacts near $13 \mathrm{~Hz}$ which then are increasingly severe until approximately $5 \mathrm{~Hz}$. The impacts cease once the frequency reduces below $2 \mathrm{~Hz}$. The responses of this non-linear system indicate that multiple solutions exist between 2-6.5 Hz. In this region the results of steady state solutions will depend on initial conditions and 
Table 1

Excitation parameter map

\begin{tabular}{lccccccccc}
\hline Run & 1 & 2 & 3 & 4 & 5 & 6 & 7 & 8 & 9 \\
\hline$T_{a}(\mathrm{Nm})$ & 2.5 & 2.5 & 2.5 & 2.75 & 2.75 & 2.75 & 3 & 3 & 3 \\
$\omega(\mathrm{rad} / \mathrm{s})$ & 56.5 & 62.8 & 69.1 & 56.5 & 62.8 & 69.1 & 56.5 & 62.8 & 69.1 \\
\hline
\end{tabular}

Table 2

Solver tolerance map

\begin{tabular}{lccccc}
\hline Case & $\mathrm{F}$ & $\mathrm{G}$ & $\mathrm{H}$ & $\mathrm{I}$ & $\mathrm{J}$ \\
\hline Relative Tolerance & $1.6 \mathrm{E}-3$ & $4 \mathrm{E}-4$ & $1 \mathrm{E}-4$ & $2.5 \mathrm{E}-5$ & $6.25 \mathrm{E}-6$ \\
Absolute Tolerance & $1.6 \mathrm{E}-6$ & $4 \mathrm{E}-7$ & $1 \mathrm{E}-7$ & $2.5 \mathrm{E}-8$ & $6.25 \mathrm{E}-9$ \\
\hline
\end{tabular}

any transient motion leading into a steady state (such as engine start or after clutch pedal disengagement) [10]. In the $6-6.5 \mathrm{~Hz}$ region there is a transition point and a simulated numerical solution will be susceptible to even a small numerical error; the system lacks stability and the rattle mode can chaotically switch between single and double-sided impacts. With this knowledge of the nature of the solutions the $9-11 \mathrm{~Hz}$ region is chosen for studying convergence. This region avoids the instability and the possibility of multiple solutions.

\subsection{Local vs. global convergence}

Numerical solutions of dynamic models for automotive rattle exhibit poor local convergence. Crossing the clearance boundary always yields some small numerical error, whether using a piecewise function or a smoothening function, where the latter is of sufficient sharpness to describe the abrupt transition. For a few boundary crossings the error is not significant, however typical simulations are of several seconds with hundreds of impacts and then the error accumulates very quickly. Two simulations run with slightly different solver tolerances will diverge and overlaid time histories could locally appear quite different (see Fig. 13 for typical time histories). Yet the global solution can be accurate. Therefore the assessment of rattle should be based on global metrics, such as the mean peak value, root-mean-square values or kurtosis. These could relate well to measured rattle signatures, which also have stochastic characteristics; given the manufacturing tolerances, other vibration sources, variation in the lubricant and so on. Once the global metrics are found to be consistent a rattle analysis tool can be used for design and analysis. For this study the RMS value for the acceleration at $\theta_{3}$ is used as the global metric for rattle intensity.

\section{Assessment of four smoothening functions}

\subsection{Design of a numerical experiment}

Solutions across an excitation parameter map (Table 1) are found and then applied to assessment of convergence. Gear's method (in this case using the Matlab algorithm ODE15s) has been selected as it is suitable for stiff systems. It uses a variable stepping algorithm so the solver tolerance is used to control time steps indirectly. For each problem formulation (non-smoothened or with a particular smoothening function) the design of experiment is performed for all runs of Table 1 for each of the solver tolerances of Table 2, in each case the mean torque is set as $T_{m}=2 \mathrm{Nm}$. The absolute tolerance set as 0.001 of the relative tolerance. Note that the relative error applies to all components of the residual vector whilst the absolute error applies to the corresponding components of the residual vector [11]. Cases (Case $\mathrm{F} \sim \mathrm{J}$ ) with decreasing tolerances are computed for convergence examination.

\subsection{Non-smoothened case and metric for assessing convergence}

The non-smoothened case is studied first to provide a baseline for assessing the smoothening functions. Simulations are run with low $\left(\xi_{1}=0.0606, \xi_{2}=0.0304, \xi_{3}=0.0077, \xi_{4}=0.0009\right.$ and $\left.\xi_{5}=0.0001\right)$ and moderate damping ratios $\left(\xi_{1}=0.0606, \xi_{2}=0.0305, \xi_{3}=0.02, \xi_{4}=0.022\right.$ and $\left.\xi_{5}=0.0265\right)$ for the higher frequency modes; those gear mesh modes are excited by the impacts. The corresponding RMS accelerations for Coordinate 3 are shown 


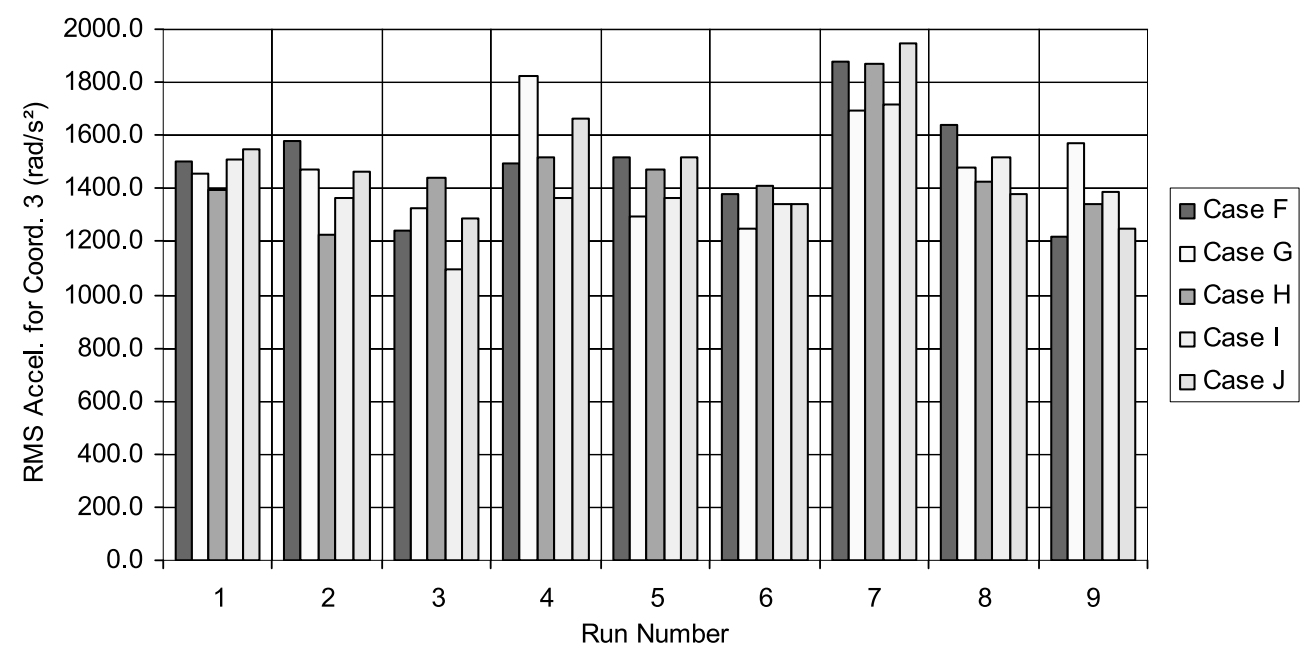

Fig. 3. RMS accelerations for Coordinate 3 with low damping ratios.

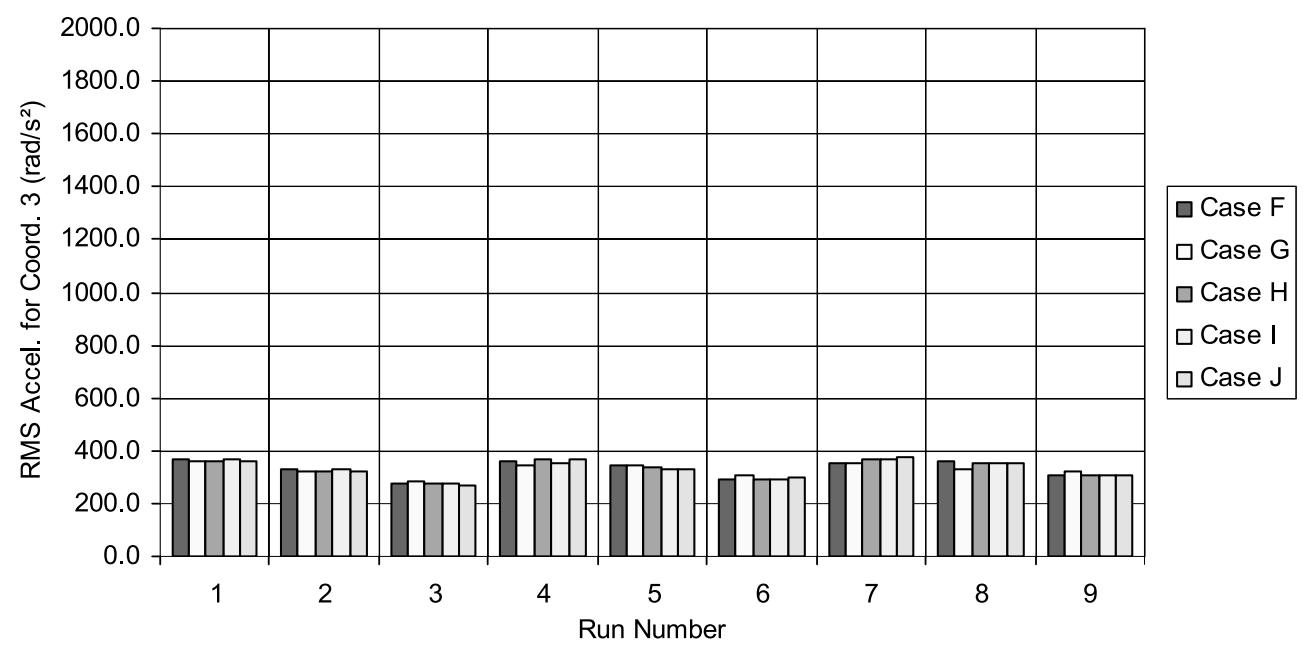

Fig. 4. RMS accelerations for Coordinate 3 with moderate damping ratios.

in the same scale for comparison in Figs 3 and 4. Inspecting the figures it is clear that the convergence is poorer with lower damping ratios; there are more impacts and thus a greater accumulation of the error from each boundary crossing. This case is selected for the subsequent studies as real-life rattle models may have more degrees of freedom with many clearances.

It is useful to quantify the result (such as shown in Fig. 3) into a single number that may be used to describe the convergence across Cases F-J. In [7] a method was proposed where an exponential function of the form $\bar{y}_{i}=A_{0}+A_{1} e^{-x_{i}}$ is first fitted to the five RMS values (Case F-J). We denote this function the "mean exponential curve". Then the standard deviation of the RMS values for each Case from the mean curve is calculated as:

$$
\hat{\sigma}_{j}=\sqrt{\frac{1}{N-1} \sum_{i=1}^{N}\left(y_{i}-\bar{y}_{i}\right)^{2}}
$$

A smaller sum for this metric signifies better convergence, here $i$ represents the case letter (Table 2 ), $N=5$ and $j$ represents the run number (Table 1). Each run then obtains a single value for $\hat{\sigma}_{j}$ as a measure of convergence. The values for $\hat{\sigma}_{j}$ may then be summed across all runs to give a value for convergence for all 45 simulations: 


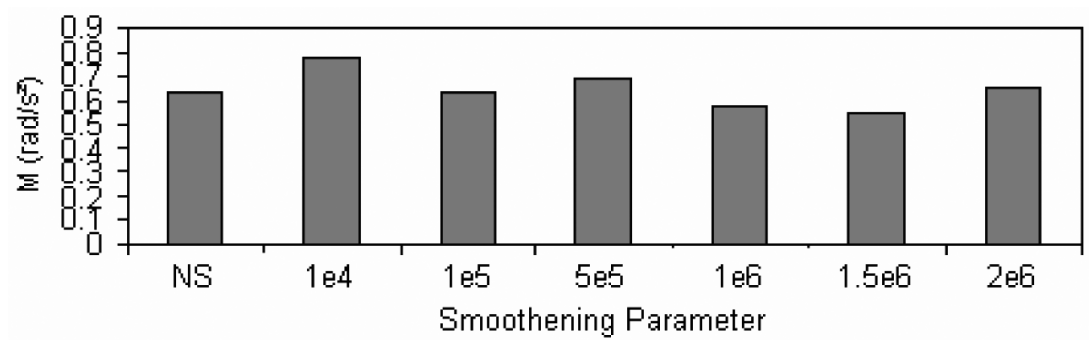

Fig. 5. $M$ for the arctan smoothing function with various smoothening parameters.

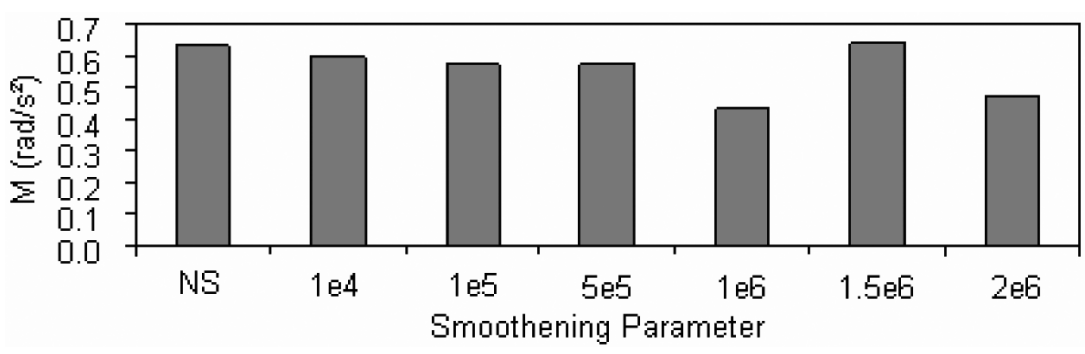

Fig. 6. $M$ for the quintic spline smoothening function with various smoothening parameters.

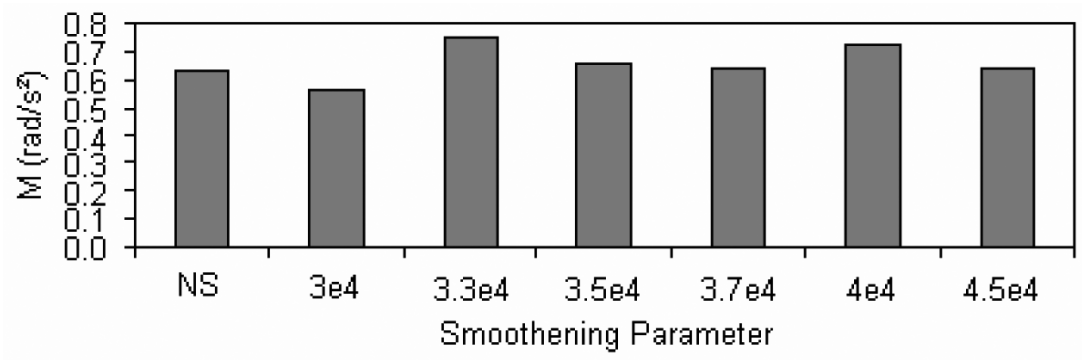

Fig. 7. $M$ for hyperbolic cos smoothening function with various smoothening parameters.

$$
M=\sum_{j=1}^{9} \hat{\sigma}_{j}
$$

The convergence metric, $M$, represents one value that provides a measure of convergence of all runs and all cases.

\subsection{Assessment of four smoothening functions}

Smoothening functions approximate the absolute function and smooth the discontinuous corner at the clearance boundary. A smoothening parameter (or regularization parameter) is used to control extent of the smoothening and a large value leads to a closer approximation of the piecewise clearance function. As follows, four smoothening functions (arctan, quintic spline, hyperbolic cos, hyperbolic tan [6]) are assessed, with the metric $M$ rating their effectiveness with respect to convergence. Figures 5-8 chart the values, including the non-smoothened case as the benchmark and a range of smoothening parameters. These figures condense results from 1125 simulations and the finding is none of the smoothing functions provide a clear benefit to the non-smoothened case. For example, with arctan smoothening (Fig. 5) using $\sigma=5 \mathrm{e} 5$ the overall convergence is slightly poorer than non-smoothened, however using $\sigma=1 \mathrm{e} 6$ the overall convergence is slightly improved. Yet when examining an overlay of the two smoothing functions there appears little difference to the abruptness of the transition (figure not shown). There is no 
Table 3

\begin{tabular}{lcc}
\hline Smoothening function & Smoothening parameter, $\sigma$ & Global Convergence Metric, $M$ \\
\hline Non-smoothened & - & 0.632 \\
Arctan & $1.5 \mathrm{e} 6$ & 0.549 \\
Quintic spline & $1 \mathrm{e} 6$ & 0.438 \\
Hyperbolic cosine & $3 \mathrm{e} 4$ & 0.564 \\
Hyperbolic tan & $1 \mathrm{e} 7$ & 0.471 \\
\hline
\end{tabular}

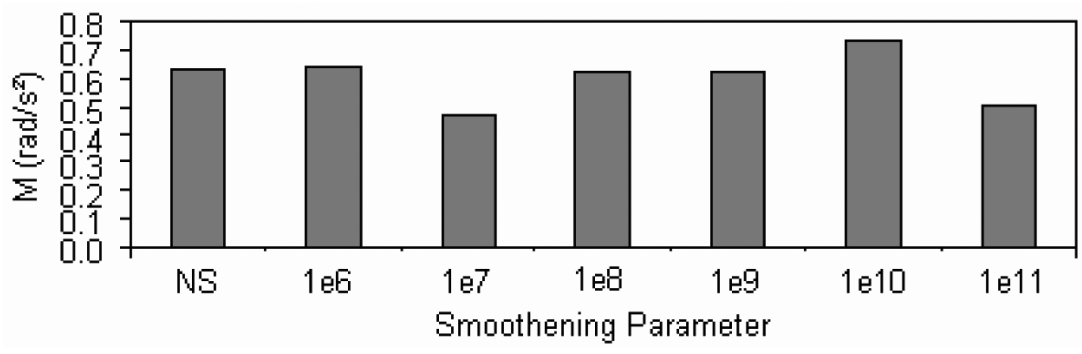

Fig. 8. $M$ for hyperbolic tan smoothening function with various smoothening parameters.

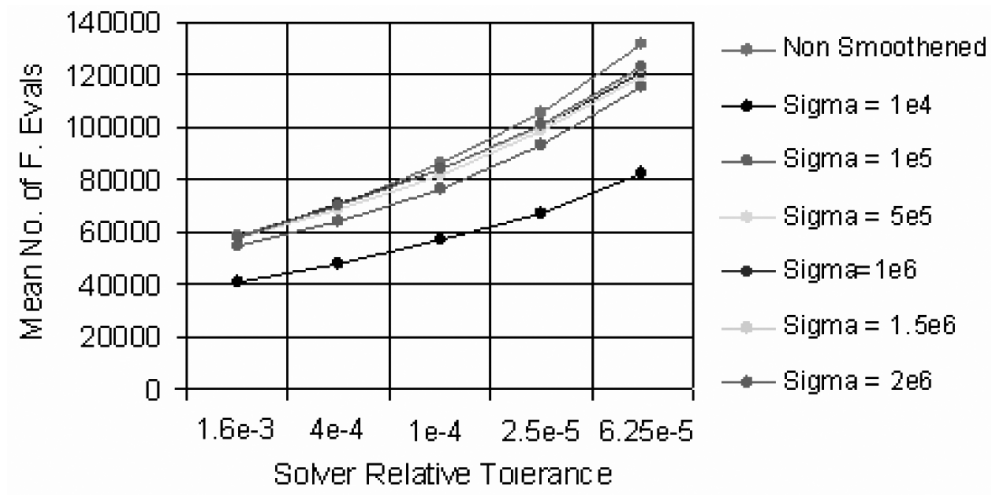

Fig. 9. Mean number of function evaluations for non-smoothened case and arctan smoothened case.

useful trend, for example, examine Fig. 8 (hyperbolic tan), and note that $\sigma=1 \mathrm{e} 9$ and $\sigma=1 \mathrm{e} 11$ both provide better convergence than $\sigma=1 \mathrm{e} 10$.

The smoothening functions and their parameters with lowest $M$ are listed in Table 3. It can be seen that with the smoothening parameters carefully chosen the convergence can be improved. Yet such careful selection would involve a similar study to this paper, an arduous exercise indeed if performed for a real-life automotive transmission rattle problem. For the particular dynamic model of Fig. 1, under the excitation map of Table 1, the quintic spline function with $\sigma=1 \mathrm{e} 9$ provides the best convergence. Based on this alone, the authors suggest this may be a reasonable function and an appropriate parameter setting that analysts could apply to similar problems. Considerations on computational efficiency follow.

\section{Performance evaluation of smoothening parameters}

\subsection{Computational efficiency study}

One of the expected advantages of smoothening functions is improved computational efficiency, which for numerical analysis can be described by the number of function evaluations. To illustrate the computation time spent, the mean number of these evaluations are quantified for all runs (Table 1) and charted against solver tolerance 


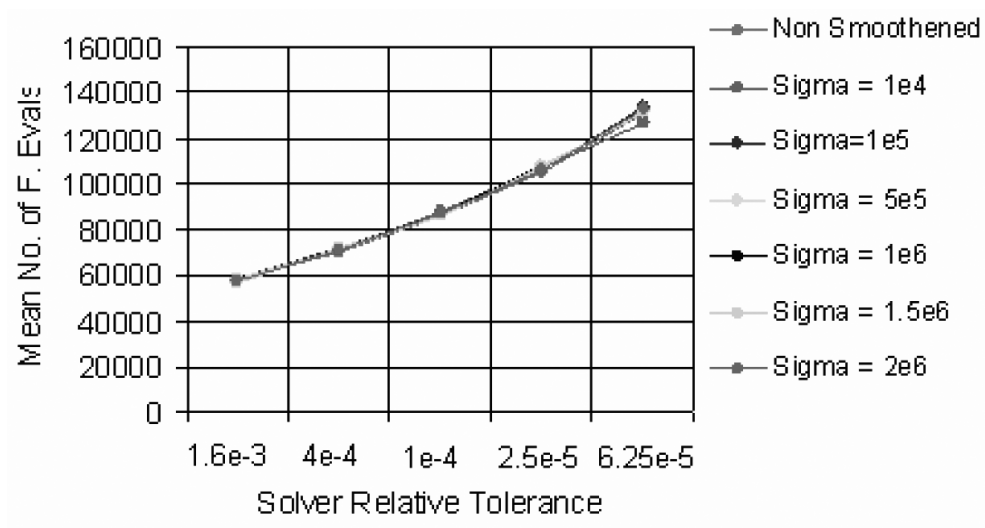

Fig. 10. Mean number of function evaluations for non-smoothened case and quintic spline smoothened case.

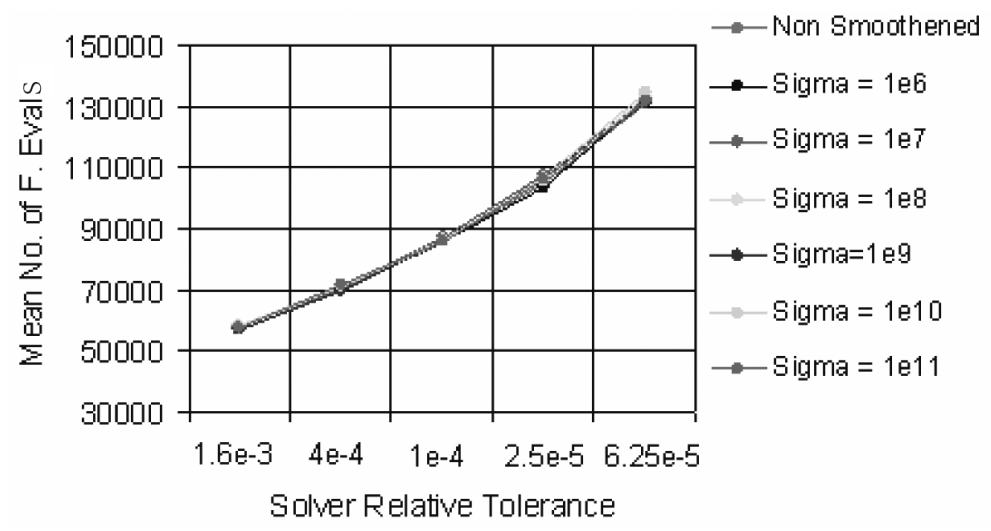

Fig. 11. Mean number of function evaluations for non-smoothened case and hyperbolic cos smoothened case.

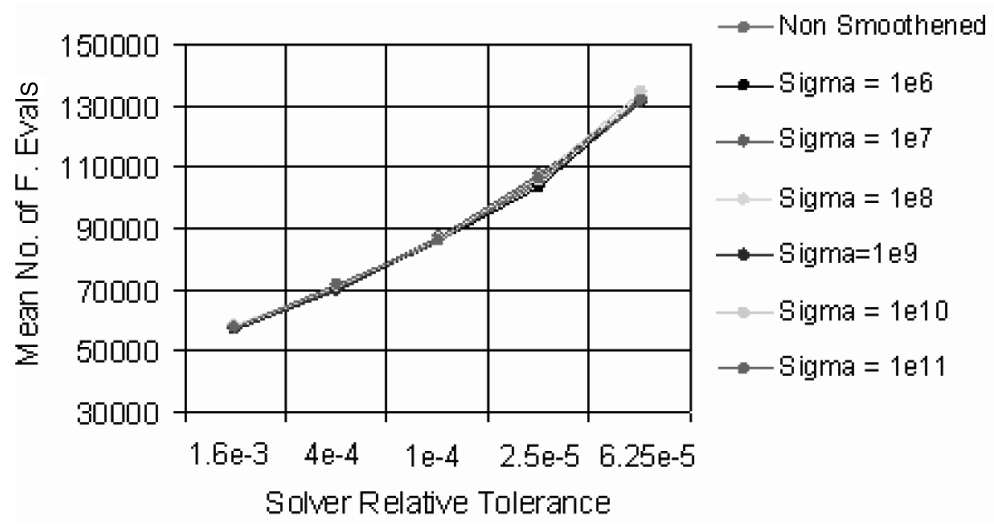

Fig. 12. Mean number of function evaluations for non-smoothened case and hyperbolic tan smoothened case.

(Table 2) in Figs 9-12. Higher mean of function evaluations leads to longer computation time by the solver, i.e. lower computation efficiency of the case. The quintic spline and hyperbolic tan functions are found to yield no improvement to the efficiency. The hyperbolic cos function provides significant improvement. Note that for the arctan function with $\sigma=1 \mathrm{e} 4$ the solutions are over-smoothened (see Section 5.2) and omitting this result the function provides only a small improvement. Naturally, the solution times increase with smaller solver tolerance. From 


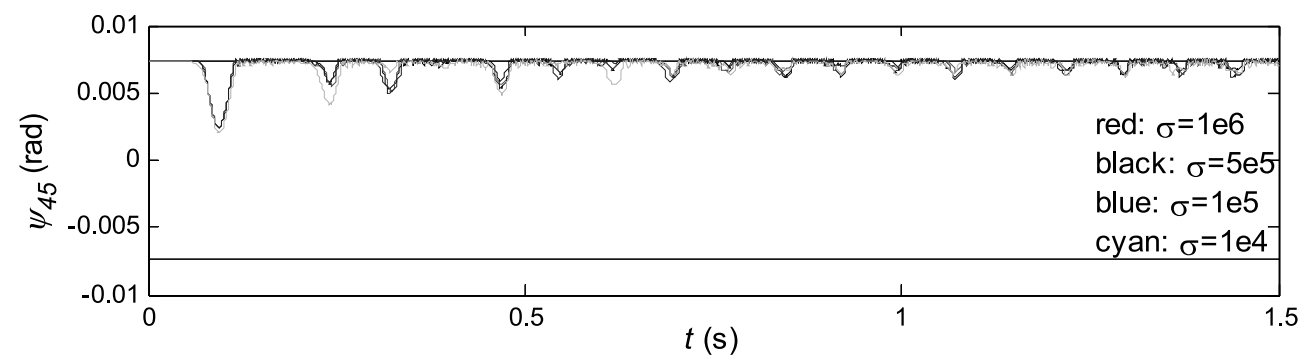

(a)

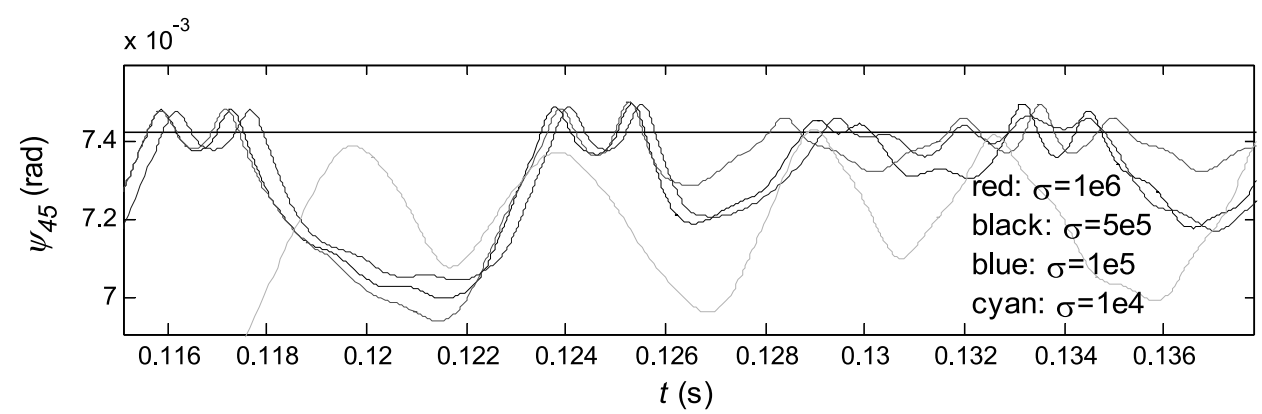

(b)

Fig. 13. Time Histories to Illustrate Over-Smoothening: a) Whole Time History; b) Magnification for a typical 0.02 s segment. Note that for $\sigma=$ le4 the boundary is not crossed and the impulsive response of higher frequencies is suppressed.

many studies the authors believe that the tolerances of Case $\mathrm{C}$ are reasonable for rattle problems where the linear system natural frequencies are less than $2000 \mathrm{~Hz}$. For Case $\mathrm{C}$ the hyperbolic cos function provides approximately $20 \%$ improvement in the computational efficiency.

\subsection{Over-smoothening}

For some smoothening parameters, the discontinuity may be over smoothened and false dynamics could be introduced. An example result with arc tan smoothening is provided in Fig. 13, where the relative angular displacement, $\Psi_{i, i+1}=\theta_{i}-\theta_{i+1}$, between Coordinates 4 and 5 is plotted, including a magnification in Fig. 13b. For this run the system is operating in the single-sided impacts mode. Where $\sigma=1 \mathrm{e} 4$ the system is over-smoothened; the clearance boundary is not crossed and the impulsive response at higher frequencies is suppressed. For $\sigma=1 \mathrm{e} 5$ the solutions could be said to be marginally over-smoothened. Such a solution overlay should be sufficient to check for over-smoothening along with the affect on rattle intensity metrics such as RMS or mean peak values.

\section{Conclusion}

A thorough examination of smoothening functions has been conducted for a generic automotive rattle problem. Methods were applied to assess the global convergence and computational efficiency of the solutions for a large set of simulations. Four smoothening functions were considered against a non-smoothened case and none were found to significantly improve convergence even when a wide range of smoothening parameters were applied. This is considered as a major limitation in current parametric space. Detailed limitations and improvements of the smoothening functions have been discussed. Our findings provide some guidelines to analysts developing automotive rattle design tools and studying similar discontinuous system problems. Each physical parameter must be carefully examined and smoothening functions and parameters should be carefully selected. As another limitation, it should be noted that there was no clear trend for setting the smoothening parameter, however some solutions can be oversmoothened and this should be avoided. In assessing the computational efficiency the hyperbolic cos smoothening 
function was found to yield a $20 \%$ improvement over non-smoothened and other smoothening functions. In this study the numerical algorithm was Gear's method with variable step size and the benefit of smoothening functions could be assessed for other solvers.

\section{References}

[1] V. Acary and B. Brogliato, Numerical Methods for Nonsmooth Dynamical Systems (Springer, 2008).

[2] V.I. Babitsky, Theory of Vibro-Impact Systems and Applications, Berlin, (Springer-Verlag, 1998).

[3] H. Zheng, Y.Y. Wang and K.P. Quek, A Refined Numerical Simulation on Dynamic Behavior of Roller Chain Drives, Shock and Vibration 11(5-6) (2004), 573-584.

[4] H. Li, B. Wen and J. Zhang, Asymptotic Method and Numerical Analysis for Self-Excited Vibration in Rolling Mill with Clearance, Shock and Vibration 8(1) (2001), 9-14.

[5] C. Padmanabhan, R.C. Barlow, T.E. Rook and R. Singh, Computational Issues Associated with Gear Rattle Analysis, Journal of Mechanical Design 117 (1995).

[6] T.C. Kim, T.E. Rook and R. Singh, Effect of Smoothening Functions on the Frequency Response of an Oscillator with Clearance Nonlinearity, Journal of Sound and Vibration 263 (2003), 665-678.

[7] A.R. Crowther, C. Halse, Z. Zhang and R. Singh, An Assessment of Smoothening Functions Applied to the Gear Rattle Model, INTERNOISE 2008, 26-29 October 2008, Shanghai, China.

[8] A.R. Crowther, C. Janello and R. Singh, Quantification of Impulsive Phenomena in Torsional Systems with Clearances, Journal of Sound and Vibration 307(3-5) (2007), 428-451.

[9] R.J. Comparin and R. Singh, Nonlinear Frequency Response Characteristics of an Impact Pair, Journal of Sound and Vibration 134(1) (1989), 259-290.

[10] R.J. Comparin and R. Singh, Frequency Response of a Multi-Degree of Freedom System with Clearances, Journal of Sound and Vibration 142(1) (1990), 101-124.

[11] MatLab 7.5.0 (R2007b) Product Help, (c) 1984-2007 The MathWorks, Inc. 

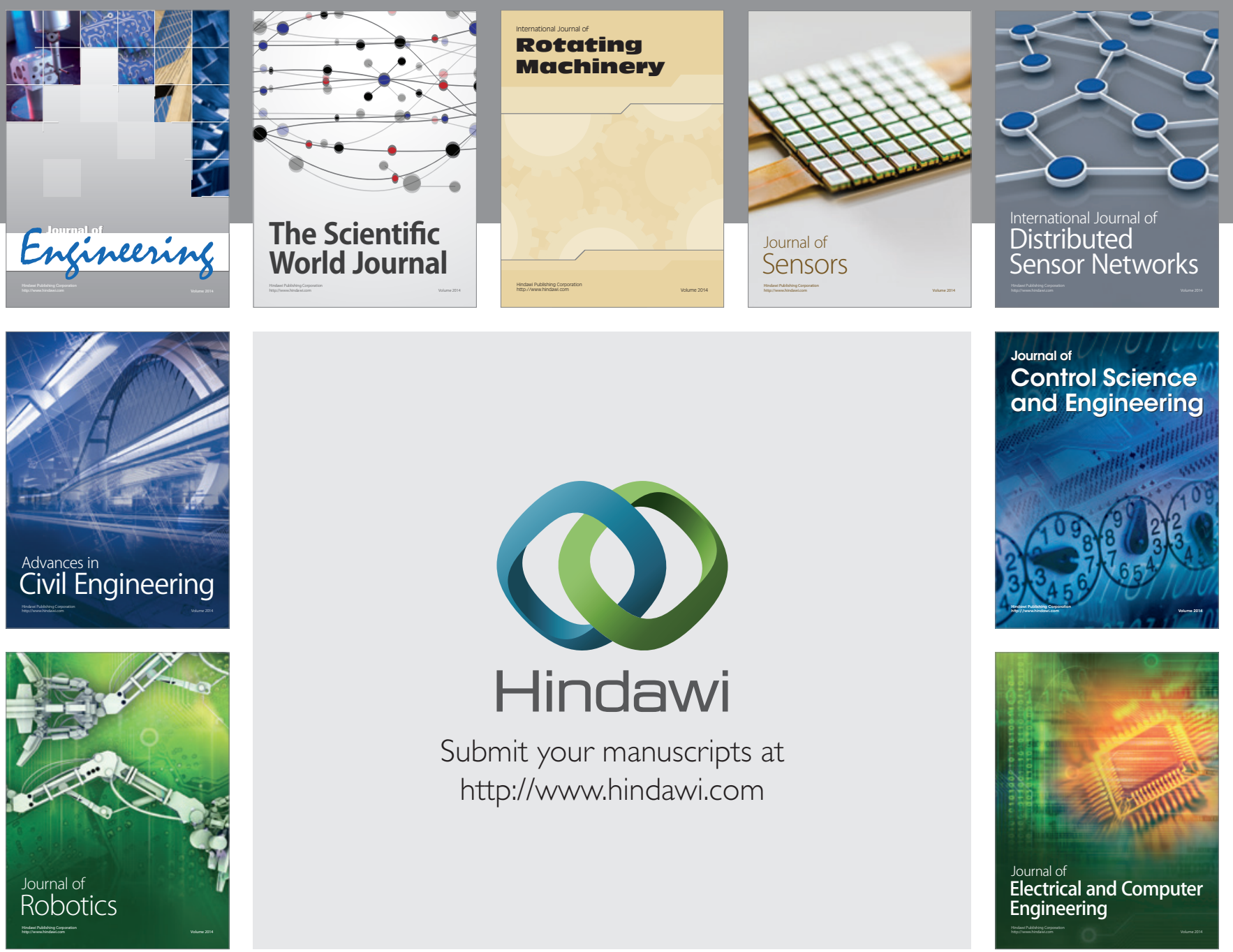

Submit your manuscripts at

http://www.hindawi.com
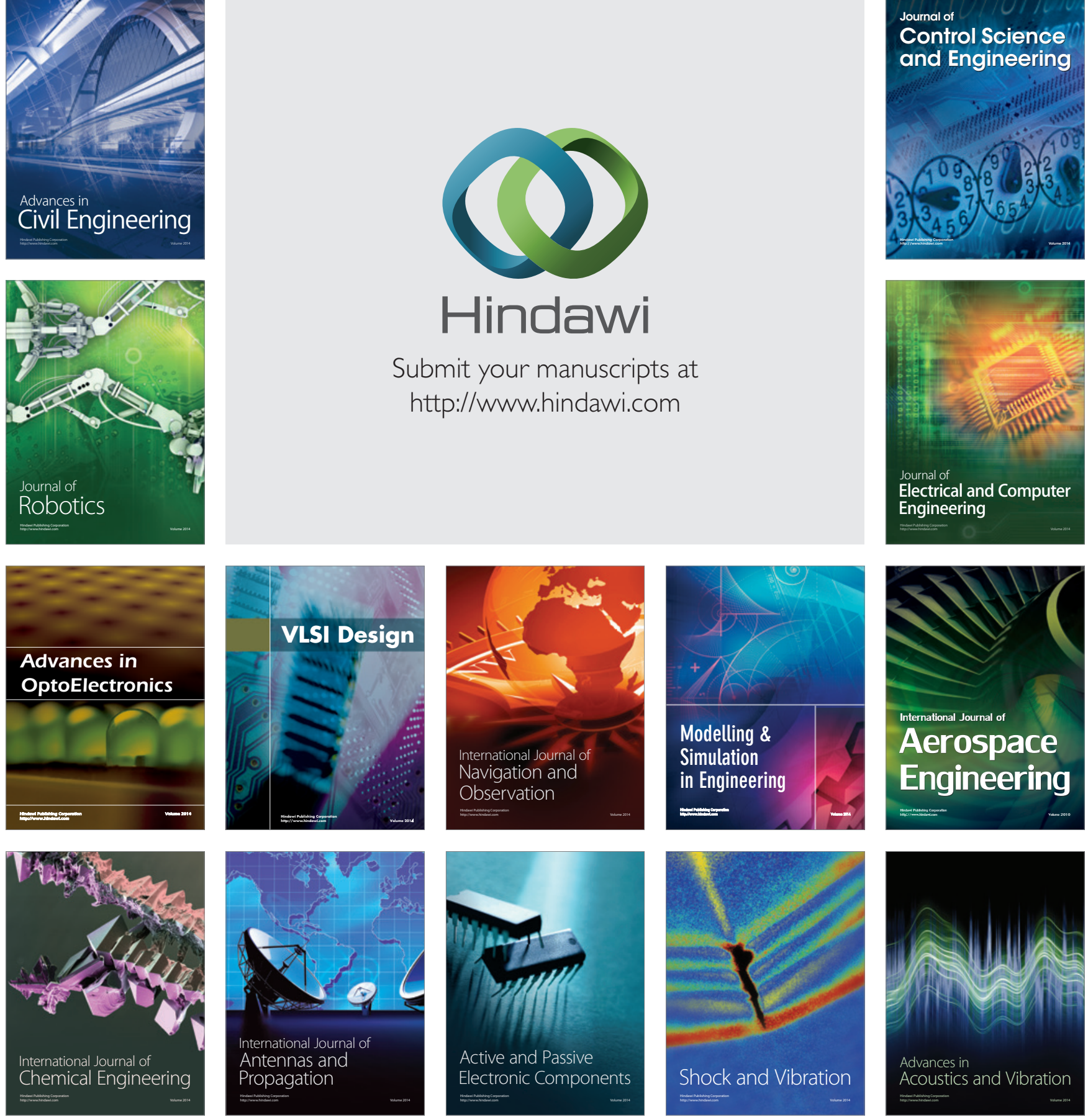